\title{
Sequential contributions to public goods
}

\author{
Hal R. Varian* \\ Department of Economics, University of Michigan, Ann Arbor, MI 48109-1220, USA
}

Received October 1991, final version received December 1992

I examine games involving private contributions to a public good and show that less of the public good will be supplied if agents move sequentially than if they move simultaneously. If the agents bid for the right to move first, the agent who values the public good least will win. If each agent chooses the rate at which he will subsidize the other agent's contributions, the subsidies that support the Lindahl allocation are the unique equilibrium outcome. I also describe two related subsidy-setting games that yield Lindahl allocations in $n$-person games with general utility functions.

\section{Introduction}

Several authors have examined the private provision of public goods in simultaneous-move games. The Nash equilibria in these games turn out to have several surprising and interesting properties. For details see Warr (1983), Bergstrom et al. (1986) and Cornes and Sandler (1986). In this paper I investigate games where agents decide on their contributions to a public good sequentially. In this sort of 'Stackelberg contribution game' the agent who moves first can credibly commit to his contribution in a way that is not possible in a simultaneous-move game.

Admati and Perry (1991) analyze a game in which agents alternate contributions to a joint project. However, in their game the project is either completed or not, and no benefits are generated from a partially completed project. In this paper, by contrast, the focus is on the amount of the public good that is provided in the contribution game.

It turns out that the ability to commit to a contribution exacerbates the free-rider problem: I show that the total amount of the public good provided in a sequential game is never larger than the amount provided in a simultaneous-move game. Along the way, I establish several other interesting

Correspondence to: H.R. Varian, Department of Economics, University of Michigan, Ann Arbor, MI 48109-1220, USA.

*This work was supported by the National Science Foundation Grant SES-8800114 and a Fulbright grant. I wish to thank Ted Bergstrom, Leif Danziger, Joel Guttman, Robert McClelland, Ig Horstman, the participants at the Michigan-Western Ontario economic theory workshop and an anonymous referee for their comments and suggestions. I also wish to thank the Santa Fe Institute for their hospitality during the period of this reasearch. 
results concerning equilibria in sequential contribution games. In particular, I examine some mechanisms in which agents choose to subsidize the contributions made by other agents, and show that these mechanisms provide simple and natural solutions to the problem of implementing Lindahl equilibria.

\section{An example with quasilinear utility}

It is instructive to start with a simple example with two agents. Each agent $i$ divides his wealth $w_{i}$ between private consumption, $x_{i} \geqq 0$, and a contribution to a public good, $g_{i} \geqq 0$. The total amount of the public good is $G=g_{1}+g_{2}$.

Each agent's utility function is linear in his private consumption and a concave increasing function of $G$, so that the utility of agent $i$ is

$$
u_{i}(G)+x_{i}=u_{i}\left(g_{1}+g_{2}\right)+w_{i}-g_{i} .
$$

Let $\bar{g}_{i}$ be the amount of the public good that maximizes agent is utility when the other agent contributes zero. We call this amount agent $i$ 's standalone contribution. We say that agent $i$ likes the public good more than agent $j$ if $\bar{g}_{i}>\bar{g}_{j}$, i.e. agent $i$ would contribute more to the public good if he were the only contributor. ${ }^{1}$ We assume that $w_{i}>\bar{g}_{i}$ so that consumption of the private good, $x_{i}$, is always strictly positive. This makes it possible to drop $w_{i}$, since it is an inessential constant in each agent's utility function.

\subsection{The reaction function}

The reaction functions and Nash equilibria for the simultaneous contribution game are reasonably well known. We sketch the derivation here for purposes of comparison with the sequential case.

First, we derive the reaction function for agent 2 . The first-order condition if agent 2 contributes a positive amount to the public good is

$$
u_{2}^{\prime}\left(g_{1}+g_{2}\right)=1 \text {. }
$$

Letting $G_{2}\left(g_{1}\right)$ be agent 2's reaction function, we must have

$$
u_{2}^{\prime}\left(g_{1}+G_{2}\left(g_{1}\right)\right)=1 \text {. }
$$

Since $\bar{g}_{2}$ is the amount that agent 2 contributes when $g_{1}=0$, we have

$$
G_{2}\left(g_{1}\right)=\bar{g}_{2}-g_{1} \text {. }
$$

The derivation is valid only when agent 2 contributes a positive amount to the public good. Since $g_{2} \geqq 0$, we must have

\footnotetext{
${ }^{1}$ It is easy to show that if one agent's marginal willingness to pay for the public good is uniformly larger than the other's, then his standalone contribution will be larger.
} 


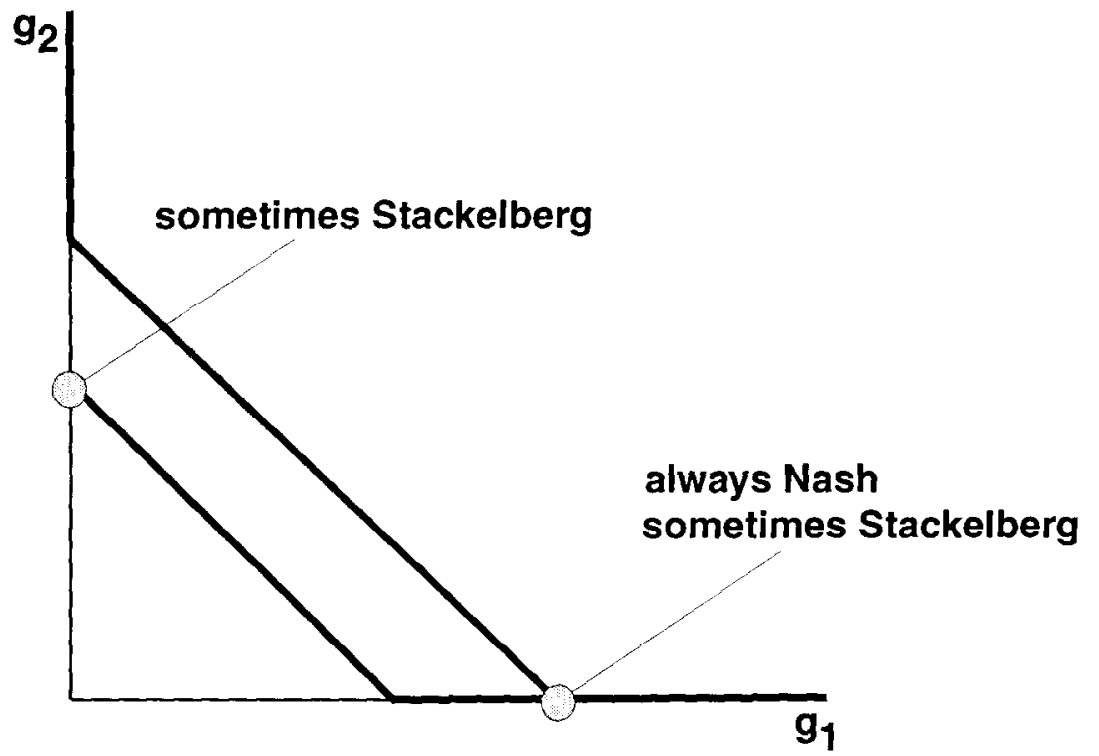

Fig. 1. Nash and Stackelberg equilibria. In the Nash equilibrium the player who likes the public good the most contributes everything and the other player free rides. In the Stackelberg equilibrium the first contributor may free ride even if he likes the public good more than the other agent.

$$
G_{2}\left(g_{1}\right)=\max \left\{\bar{g}_{2}-g_{1}, 0\right\} .
$$

This 'kink' in the reaction function is what makes the analysis interesting.

\subsection{The Nash equilibrium}

A Nash equilibrium is a pair of contributions $\left(g_{1}, g_{2}\right)$ such that

$$
g_{1}=G_{1}\left(g_{2}\right) \text { and } g_{2}=G_{2}\left(g_{1}\right) \text {. }
$$

A Nash equilibrium is depicted in fig. 1. In the case depicted, agent 1 likes the public good more than agent 2 . In this case agent 1 contributes the entire amount of the public good and agent 2 free rides. If both agents have the same tastes for the public good, the reaction functions overlap and there is a whole range of equilibrium contributions, although there still is a unique equilibrium amount of the public good.

\subsection{The Stackelberg equilibrium}

We assume that agent 1 moves first. The utility of agent 1 as a function of his contribution is given by

$$
V_{1}\left(g_{1}\right)=u_{1}\left(g_{1}+G_{2}\left(g_{1}\right)\right)-g_{1}
$$




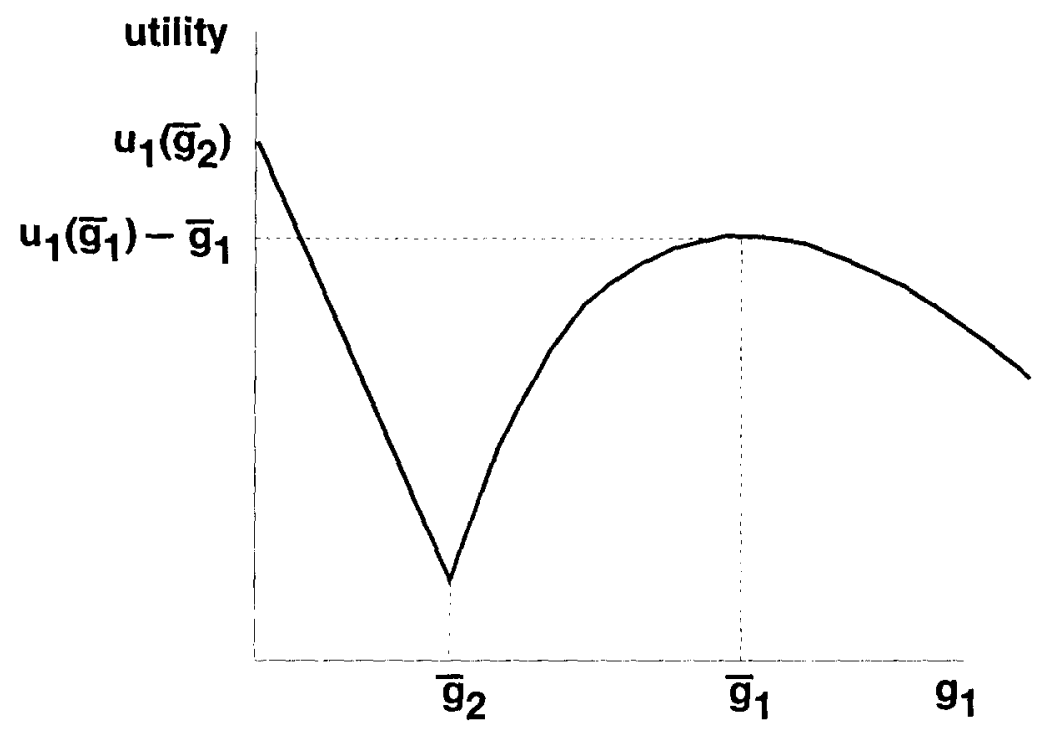

Fig. 2. Utility of the first contributor as a function of his gift. In this example, agent I's optimal choice is to contribute zero, but if the 'hump' were higher, he would want to contribute $\bar{g}_{1}$.

$$
=u_{1}\left(g_{1}+\max \left\{\bar{g}_{2}-g_{1}, 0\right\}\right)-g_{1} \text {. }
$$

We can also write this as

$$
V_{1}\left(g_{1}\right)= \begin{cases}u_{1}\left(\bar{g}_{2}\right)-g_{1} & \text { for } g_{1} \leqq \bar{g}_{2} \\ u_{1}\left(g_{1}\right)-g_{1} & \text { for } g_{1} \geqq \bar{g}_{2}\end{cases}
$$

It is clear from fig. 2 that there are two possible optima: either the first agent contributes zero and achieves utility $u_{1}\left(\bar{g}_{2}\right)$ or he contributes $\bar{g}_{1}$ and achieves utility $u_{1}\left(\bar{g}_{1}\right)-\bar{g}_{1}$.

Case 1. The agent who likes the good least is the first contributor. In this case the optimal choice by the first player is to contribute zero. This is true since

$$
u_{1}\left(\vec{g}_{2}\right)>u_{1}\left(\bar{g}_{1}\right)>u_{1}\left(\bar{g}_{1}\right)-\bar{g}_{1} .
$$

Case 2. The agent who likes the public good the most is the first contributor. In this case, either contributor may free ride. The easiest way to see this is by example. Suppose that agent $i$ 's utility for the public good is $u_{i}(G)=a_{i} \ln G$, so that agent $i$ 's standalone contribution is $a_{i}$. Then the first contributor will get utility $\ln a_{1}-a_{1}$ or $\ln a_{2}$, and either of these may be larger. 
If the agents have tastes that are very similar, then the first contributor will free ride on the second's contribution. However, if the first agent likes the public good much more than the second, then the first agent may prefer to contribute the entire amount of the public good himself.

Referring to fig. 1 we see that there are two possible Stackelberg equilibria: one is the Nash equilibrium, in which the agent who likes the good most contributes everything. The other Stackelberg equilibrium is where the agent who likes the good least contributes everything. This equilibrium cannot arise as a Nash equilibrium since the threat to free ride by the agent who likes the public good most is not credible in the simultaneous-move game.

Note that it is always advantageous to move first since there are only two possible outcomes and the first mover gets to pick the one he prefers. Also note that the sum of the utilities is higher at the higher level of the public good. It follows that if you want to ensure that the higher level of the public good is provided, then you should make sure that the person who likes the good least moves first.

\section{Examples}

Here we briefly describe two examples of free riding in games involving sequential contributions to public goods. The first is a variation on the Samaritan's dilemma as described by Buchanan (1975), Varian (1982), and Lindbeck and Weibull (1988). Consider a game between the young generation and the old generation. The old generation has two choices: they can save for their retirement, or they can squander their earnings on their consumption. The young generation likewise has two strategies: they can support the older generation when it retires, or they can let them starve.

A natural assignment of payoffs to these strategies implies that there are two Nash equilibria in this game. In one equilibrium, the older generation expects the younger generation to support them and they therefore choose to squander. Given the choice between letting the older generation starve or providing support, the younger generation chooses to provide support. In the other Nash equilibrium, the older generation expects the younger generation not to provide support, and so they prudently choose to save. Since they reach retirement with adequate resources, the younger generation chooses not to support them.

However, only one of these equilibria survives when we take account of the fact that the older generation gets to move first. The unique subgame perfect equilibrium is for the older generation to squander, recognizing that the younger generation will be forced to provide for them in their old age. Even though the older generation cares more about its consumption that the younger generation does, the older generation still finds it optimal to free ride on the younger generation's contribution. 
The second example has to do with reproductive strategy in animals. Males and females normally contribute equal amounts of genetic material to their offspring. However, they often contribute unequally to the care of the offspring. The degree to which males and females provide care for their offspring depends, in part, on the timing of their choices. In mammals, the male is generally the first mover when he fertilizes the eggs of the female. Once the female egg is fertilized the male may well depart, leaving most childcare responsibilities to the female.

In some species of fish, the childcare responsibilities are completely reversed. Among fish, the female is the first mover when she lays the eggs. The male moves second by fertilizing them. At that point the female may well depart, leaving the male to care for the eggs.

Forsyth (1986) describes a particularly interesting case in which there is yet another level of free riding. There are two type of bluegill sunfish: large 'territorial' males and much smaller 'satellite' males. The territorial males build nests in order to attract females, while the satellite males hover around the nest built by the territorial male. After a female selects a nest and releases her eggs, the satellite male rushes into the nest and releases its sperm at the same time as the territorial male attempts to fertilize the eggs. The territorial male then has no recourse but to care for the eggs, even though only a fraction of them carry his genetic material, since he is better able to fend off predators than the smaller satellite male. The female bluegill and the satellite male then blithely swim away, free riding on the childcare activities of the territorial male!

\section{Bidding for the right to move first}

Since the first mover always has an advantage in the sequential contribution game, we might consider auctioning off the right to move first. If the first mover likes the public good much more than the second, then he will provide the entire amount of the public good anyway, so it is no advantage to him to be the first mover. The advantage to the first mover only arises when the players have similar tastes for the public good. In this case, each player would prefer to move first and free ride on the other's contribution.

Consider, then, the case where agent 1 likes the public good a bit more than agent 2 , so that $\bar{g}_{1}>\bar{g}_{2}$, but not so much more as to contribute everything himself. Agent 1 can get utility $u_{1}\left(\bar{g}_{2}\right)$ by moving first and free riding. If he moves second, he gets utility $u_{1}\left(\bar{g}_{1}\right)-\bar{g}_{1}$. The amount that he would be willing to bid to move first, $b_{1}$, is therefore

$$
b_{1}=u_{1}\left(\bar{g}_{2}\right)-u_{1}\left(\bar{g}_{1}\right)+\bar{g}_{1} .
$$

We assume that the bids are either thrown away or given to the other agent. 
Since there are no income effects, it does not matter what is done with the bids.

The difference between the bids of the two agents is

$$
b_{1}-b_{2}=\left[u_{1}\left(\bar{g}_{2}\right)-u_{1}\left(\bar{g}_{1}\right)\right]+\left[u_{2}\left(\bar{g}_{2}\right)-u_{2}\left(\bar{g}_{1}\right)\right]-\left[\bar{g}_{2}-\bar{g}_{1}\right] .
$$

It seems plausible to suppose that the agent who values the public good more would be willing to pay more to move first. However, this is exactly wrong! Under our assumptions, the agent who values the public good least is willing to pay more for the first-mover position.

To see this, note that concavity of the utility functions gives us the following inequalities:

$$
\begin{aligned}
& u_{1}\left(\bar{g}_{2}\right)-u_{1}\left(\bar{g}_{1}\right) \leqq u_{1}^{\prime}\left(\bar{g}_{1}\right)\left[\bar{g}_{2}-\bar{g}_{1}\right] . \\
& u_{2}\left(\bar{g}_{2}\right)-u_{2}\left(\bar{g}_{1}\right) \leqq u_{2}^{\prime}\left(\bar{g}_{1}\right)\left[\bar{g}_{2}-\bar{g}_{1}\right] .
\end{aligned}
$$

Substituting these into (1) we have

$$
b_{1}-b_{2} \leqq\left[u_{1}^{\prime}\left(\bar{g}_{1}\right)+u_{2}^{\prime}\left(\bar{g}_{1}\right)-1\right]\left(\bar{g}_{2}-\bar{g}_{1}\right) .
$$

Since $u_{1}^{\prime}\left(\bar{g}_{1}\right)=1$, this simplifies to

$$
b_{1}-b_{2} \leqq u_{2}^{\prime}\left(\bar{g}_{2}\right)\left[\bar{g}_{2}-\bar{g}_{1}\right]<0,
$$

where the least inequality follows since $\bar{g}_{1}>\vec{g}_{2}$.

It follows that $b_{1}<b_{2}$. That is, the agent who likes the public good the least will be willing to pay the most in order to move first. As we have seen, this will ensure that the largest amount of the public good will be provided. Essentially each agent is bidding for the right to free ride on the other agent, and it is better to free ride on someone who will provide a lot of the public good than someone who will provide only a little.

\section{Subsidizing the other agent}

In the game considered above, one agent is able to commit to a contribution to the public good before the other agent makes his choice. The contribution by the first agent affects the benefits that the second agent receives from his contribution. In this section we examine public goods games in which the agents can influence the cost to other agents of their contributions. In particular, we examine what will happen if one agent has the opportunity to subsidize the other agent's contributions.

For simplicity we consider the case of identical utilities. As we have seen, the Nash equilibrium amount of the public good, $G^{\mathbf{n}}$, is determined by the condition 


$$
u_{1}^{\prime}\left(G^{\mathrm{n}}\right)=1
$$

and any set of contributions $\left(g_{1}^{\mathrm{n}}, g_{2}^{\mathrm{n}}\right)$ such that $g_{1}^{\mathrm{n}}+g_{2}^{\mathrm{n}}=G^{\mathrm{n}}$ is a Nash equilibrium.

Suppose that agent 1 offers to subsidize agent 2's contributions at the rate $s_{2}$. The payoffs to the two agents become

$$
\begin{aligned}
& u_{1}\left(g_{1}+g_{2}\right)-g_{1}-s_{2} g_{2}, \\
& u_{2}\left(g_{1}+g_{2}\right)-\left(1-s_{2}\right) g_{2} .
\end{aligned}
$$

Since agent 2 now faces a lower cost of contributing than agent 1 , agent 2 will contribute the entire amount of the public good. The amount that agent 2 contributes, $G\left(s_{2}\right)$, is determined by the equation

$$
u_{2}^{\prime}\left(G\left(s_{2}\right)\right)=1-s_{2} \text {. }
$$

Given our assumption that $u_{2}(G)$ is strictly concave, $G\left(s_{2}\right)$ will be a continuous increasing function. Agent 1's utility from offering the subsidy rate $s_{2}$ is

$$
u_{1}\left(G\left(s_{2}\right)\right)-s_{2} G\left(s_{2}\right) .
$$

Originally agent 1 had utility $u_{1}\left(G^{\mathrm{n}}\right)-g_{1}^{\mathrm{n}}=u_{1}(G(0))-g_{1}^{\mathrm{n}}$. The increase in agent 1 's utility from offering the subsidy is

$$
u_{1}\left(G\left(s_{2}\right)\right)-u_{1}(G(0))+g_{1}^{n}-s_{2} G\left(s_{2}\right) \text {. }
$$

As $s_{2}$ approaches zero, this expression converges to $g_{1}^{n} \geqq 0$. This implies that if agent 1 was initially contributing a positive amount, he would be strictly better off by offering to subsidize agent 2's contribution by a sufficiently small amount. Intuitively, if agent 1 offers a very tiny subsidy to agent 2 , agent 2 will end up contributing the entire amount of the public good in the second stage. But a tiny subsidy hardly costs agent 1 anything, which means that each agent always will want to subsidize the other agent's contribution.

Roberts (1987) and Bergstrom (1989) show that if a subsidy on contributions to a public good is financed by an equal lump-sum tax, then each agent prefers that the other agent be subsidized. We have shown that each agent prefers to subsidize the other agent even if he must pay for the subsidy himself. However, the Roberts-Bergstrom result holds for general preferences, while our result only holds for quasilinear preferences.

\section{Equilibrium subsidies}

We have seen that each agent will prefer to subsidize the other agent in 
our contribution game. Suppose that each agent simultaneously names a rate at which he is willing to subsidize the other agent. Then, given these subsidy rates, the agents play a simultaneous contribution game. What is the equilibrium of this game?

In order to answer this question, we need some facts about the Lindhal allocation in this model. In the case of quasilinear utility there will be a unique amount of the public good that maximizes the sum of the utilities. This amount, $G^{\mathrm{e}}$, satifies the first-order condition

$$
u_{1}^{\prime}\left(G^{\mathrm{e}}\right)+u_{2}^{\prime}\left(G^{\mathrm{e}}\right)=1
$$

This is just the familiar condition that the sum of the marginal willingessesto-pay must equal marginal cost.

Suppose that we choose rates $s_{i}^{\mathrm{e}}$ to support the efficient amount of the public good:

$$
u_{1}^{\prime}\left(G^{\mathrm{e}}\right)=1-s_{1}^{\mathrm{e}} ; \quad u_{2}^{\prime}\left(G^{\mathrm{e}}\right)=1-s_{2}^{\mathrm{e}},
$$

Note that (3) and (4) together imply

$$
s_{1}^{e}+s_{2}^{e}=1 \text {. }
$$

Eq. (5) implies that the utility of agent 1 at the Lindahl allocation is

$$
u_{1}\left(G^{\mathrm{e}}\right)-\left(1-s_{1}^{\mathrm{e}}\right) g_{1}-s_{2}^{\mathrm{e}} g_{2}=u_{1}\left(G^{\mathrm{e}}\right)-\left(1-s_{1}^{\mathrm{e}}\right) G^{\mathrm{e}} .
$$

Hence $s_{2}^{\mathrm{e}}=1-s_{1}^{\mathrm{e}}$ is effectively a Lindahl price for agent 1 ; accordingly, we call $\left(s_{1}^{\mathrm{e}}, s_{2}^{\mathbf{e}}\right)$ the Lindahl subsidies.

Theorem 1. The unique subgame perfect equilibrium of the subsidy-setting game yields the Lindahl subsidies, and the resulting allocation is the Lindahl allocation.

Proof. If we have an interior equilibrium at the contribution stage, the amount of the public good must satisfy

$$
u_{1}^{\prime}\left(g_{1}^{*}+g_{2}^{*}\right)=1-s_{1} ; \quad u_{2}^{\prime}\left(g_{1}^{*}+g_{2}^{*}\right)=1-s_{2} \text {. }
$$

Suppose that agent 2, say, is not contributing. There is no cost to agent 1 of increasing $s_{2}$ up to the point where agent 2 is just on the verge of contributing. We assume that this has been done, which means that (6) will apply even in the case of boundary solutions.

If agent 1 slightly increases the subsidy agent 2 faces, then agent 2 will do 
all the contributing, and agent 1 will contribute zero. If we are in equilibrium, such a change cannot benefit agent 1 . Using a limiting argument similar to that used to establish eq. (2) this implies

$$
u_{1}\left(g_{1}^{*}+g_{2}^{*}\right)-\left(1-s_{1}\right) g_{1}^{*}-s_{2} g_{2}^{*} \geqq u_{1}\left(g_{1}^{*}+g_{2}^{*}\right)-s_{2}\left(g_{1}^{*}+g_{2}^{*}\right) .
$$

After simplification, we have

$$
g_{1}^{*}\left(s_{1}+s_{2}-1\right) \geqq 0 .
$$

Similarly, if agent 1 reduces the subsidy rate that agent 2 faces, then agent 1 will do all the contributing. In equilibrium agent 1 cannot benefit from this change which implies

$$
u_{1}\left(g_{1}^{*}+g_{2}^{*}\right)-\left(1-s_{1}\right) g_{1}^{*}-s_{2} g_{2}^{*} \geqq u_{1}\left(g_{1}^{*}+g_{2}^{*}\right)-\left(1-s_{1}\right)\left(g_{1}^{*}+g_{2}^{*}\right) .
$$

Simplification yields

$$
g_{2}^{*}\left(s_{1}+s_{2}-1\right) \leqq 0 .
$$

The same arguments applied to agent 2 yield

$$
\begin{aligned}
& g_{2}^{*}\left(s_{1}+s_{2}-1\right) \geqq 0, \\
& g_{1}^{*}\left(s_{1}+s_{2}-1\right) \leqq 0 .
\end{aligned}
$$

At least one of $\left(g_{1}^{*}, g_{2}^{*}\right)$ must be non-zero in equilibrium. This observation, together with (7)-(10), implies $s_{1}+s_{2}=1$. It follows from (6) that the resulting allocation is the Lindahl allocation.

The intuition behind this result is rather nice: if agent 1 contributes directly to the public good, it costs him a dollar for each dollar he contributes. But if he contributes indirectly, say by giving a 10 percent subsidy, then it costs agent 1 only ten cents for each dollar that agent 2 contributes to the public good. Each agent will continue to subsidize the other until the marginal cost of contributing directly is equal to the marginal cost of contributing indirectly. In the case of identical consumers, this is where each agent gives the other a 50 percent subsidy - which is the Lindahl price.

\section{Related literature}

There is a large literature on designing mechanisms to solve the public goods problem. Groves (1979), Groves and Ledyard (1987), Moore (1991), Ledyard (1992) provide surveys of various aspects of this literature. 
The classic solutions to the free-rider problem are due to Groves and Ledyard (1977), Hurwicz (1979), and Walker (1981). However, the most closely related result to ours is Guttman $(1978,1987)$. In the first stage of Guttman's game each agent announces a rate at which he will match the other agent's contribution. In the second stage each agent contributes a 'flat' amount plus a matching amount tied to the other agent's direct contribution. Guttman argues that the equilibrium of this game is the Pareto-efficient amount of the public good. ${ }^{2}$ In Guttman's game, the matching contribution goes directly to the public good; in my game, the subsidy is paid to the other contributor. However, the strategic nature of the two games is similar since each agent is effectively setting a price for the other agent's contribution. Guttman (1986) describes some experimental evidence in his matching game.

More recently, Jackson and Moulin (1992) describe a two-stage bidding game that yields a Lindahl-like allocation in the case of a discrete public good. In the first stage of the Jackson-Moulin game, each agent announces the total value of the public good. In the second stage, each agent announces his own valuation. The Jackson-Moulin game implements an efficient allocation in undominated Nash equilibrium, although they offer a variation that implements efficiency in subgame perfect equilibrium.

In Varian (1989) I describe a subsidy-setting game for implementing Lindahl-like outcomes for completely general externalities problems. The mechanism in Varian (1989) is more general than the subsidy-setting game described here, but it is also more complicated. The nice thing about the subsidy game is that it is very natural.

\section{General utility functions}

I have described several results for public goods problems with two stages in which a price or a quantity is set in the first stage, and further quantity choices are made in the second stage. However, the quasilinear case is very special: do these results generalize to a model with income effects?

Let $u_{i}\left(G, x_{i}\right)$ be a utility function with $G$ the level of the public good and $x_{i}$ the private consumption of agent $i$. As before, I assume that utility is a differentiable, strictly concave function.

I first derive the form of the reaction function. Agent 2's maximization problem is

$$
\max _{x_{2}, g_{2}} u_{2}\left(g_{1}+g_{2}, x_{2}\right)
$$

${ }^{2}$ Guttman (1978) did not contain a complete proof of this result because of space limitations. 
such that $g_{2}+x_{2}=w_{2}$,

$$
g_{2} \geqq 0 \text {. }
$$

We can add $g_{1}$ to each side of the constraints and use the definition $G=g_{1}+g_{2}$ to write the problem as

$$
\max _{x_{2}, G} u_{2}\left(G, x_{2}\right)
$$

such that $G+x_{2}=w_{2}+g_{1}$,

$$
G \geqq g_{1} \text {. }
$$

In this problem, agent 2 is choosing the level of the public good, subject to the constraint that the level that he chooses is at least as large as the contribution of agent 1 .

Following Bergstrom et al. (1986) we note that this problem is a standard consumer demand problem except for the inequality constraint. Let $f_{2}(w)$ be agent 2's demand function for the public good. It follows from the above remarks that

$$
G=g_{1}+g_{2}=\max \left\{f_{2}\left(w_{2}+g_{1}\right), g_{1}\right\} .
$$

Subtracting $g_{1}$ from each side of this equation, we have the reaction function:

$$
G_{2}\left(g_{1}\right)=\max \left\{f_{2}\left(w_{2}+g_{1}\right)-g_{1}, 0\right\} .
$$

According to this reaction function, agent 1 will either contribute zero or the amount of the public good that he would demand if his wealth were $w_{1}+g_{2}$ minus the amount contributed by the other agent. The following assumption is quite natural:

Normal goods. Both the public and the private good are strictly normal goods at all levels of wealth.

Given this assumption it is easy to see the general shape of the reaction function. When $g_{1}=0$, agent 2 will contribute $f_{2}\left(w_{2}\right)$. As $g_{1}$ increases, the contribution of agent 2 will decrease, but less than one-for-one. For some $g_{1}^{c}$ we may have $f_{2}\left(w_{2}+g_{1}^{\mathrm{c}}\right)=0$; at this point agent 1 contributes so much that agent 2 chooses to free ride. We call this amount the complete crowding out contribution.

We summarize some properties of the reaction function in the following fact, the proof of which follows immediately from the assumption.

Fact 1 . The reaction function $G_{2}\left(g_{1}\right)$ is a non-increasing function. It will be 
strictly decreasing when it is not equal to zero. The function $H\left(g_{1}\right)=$ $g_{1}+G_{2}\left(g_{1}\right)$ is a strictly increasing function.

As before, we can use this reaction function to calculate the Nash equilibria and the Stackelberg equilibrium. A Nash equilibrium is a solution $\left(g_{1}^{\mathrm{n}}, g_{2}^{\mathrm{n}}\right)$ to

$$
\begin{aligned}
& g_{1}^{\mathrm{n}}=\max \left\{f_{1}\left(w_{1}+g_{2}^{\mathrm{n}}\right)-g_{2}^{\mathrm{n}}, 0\right\}, \\
& g_{2}^{\mathrm{n}}=\max \left\{f_{2}\left(w_{2}+g_{1}^{\mathrm{n}}\right)-g_{1}^{\mathrm{n}}, 0\right\} .
\end{aligned}
$$

A Stackelberg equilibrium is a pair $\left(g_{1}^{\mathrm{s}}, G_{2}\left(g_{1}^{\mathrm{s}}\right)\right)$ for which $g_{1}^{\mathrm{s}}$ solves

$$
\max _{g_{1}} u_{1}\left(g_{1}+\max \left\{f_{2}\left(w_{2}+g_{1}\right)-g_{1}, 0\right\}, w_{1}-g_{1}\right) .
$$

We want to compare the solutions of these two sets of equations. This comparison is made simpler by noting that Bergstrom et al. (1986) have proved that under the normality assumption we have made there is a unique Nash equilibrium. There will also be one Stackelberg equilibrium for each ordering of the agents.

\section{Results for general utility functions}

We have three sets of results. The first set of resuits concerns who contributes and who free rides. The second set of results concerns the effect of redistributions of wealth. The third set of results concerns how the amount of the public good provided in the Stackelberg equilibrium comparcs with the amount provided in the Nash equilibrium.

\subsection{Free riding}

Fact 2. If the standalone contribution is less than the complete crowding out contribution $\left(\bar{g}_{1}<g_{1}^{\mathrm{c}}\right)$, then both agents must contribute in the Stackelberg equilibrium.

Proof. Evaluate the right derivative of agent 1's utility function at $g_{1}^{\text {c. We }}$ have

$$
\frac{\partial u_{1}\left(g_{1}^{\mathrm{c}}, w_{1}-g_{1}^{\mathrm{c}}\right)}{\partial G}-\frac{\partial u_{1}\left(g_{1}^{\mathrm{c}}, w_{1}-g_{1}^{\mathrm{c}}\right)}{\partial x_{1}}<0
$$

The inequality follows since the derivative equals zero at $\bar{g}_{1}$, and $g_{1}^{\text {c }}>\bar{g}_{1}$. [Recall that $u_{1}\left(g_{1}, w_{1}-g_{1}\right)$ is a concave function]. It follows that agent 1's utility will increase if he contributes less than $g_{1}^{\mathfrak{c}}$, even if he is the only one to 
contribute. The fact that the other agent will also contribute can only increase the first agent's utility. Hence the Stackelberg equilibrium must involve contributions by both agents.

Fact 3. If there is a Nash equilibrium with $g_{1}^{\mathrm{n}}=0$, then this is also a Stackelberg equilibrium.

Proof. By definition of a Nash equilibrium, agent 2 is on his reaction curve, so we only need to show that agent 1 is on his reaction curve. If agent 1 contributes 0 , then agent 2 will contribute $\bar{g}_{2}$. Let $g_{1}>0$ be any other possible contribution by agent 1 . Then we have

$$
u_{1}\left(\bar{g}_{2}, w_{1}\right)>u_{1}\left(g_{1}+\bar{g}_{2}, w_{1}-g_{1}\right)>u_{1}\left(g_{1}+G_{2}\left(g_{1}\right), w_{1}-g_{1}\right) .
$$

The first inequality follows from the Nash assumption. The second inequality follows since $G_{2}(0)=\bar{g}_{2}$ and $G_{2}\left(g_{1}\right)$ is a non-increasing function.

\subsection{Wealth redistribution}

Fact 4. Suppose that we have a Stackelberg equilibrium $\left(g_{1}^{\mathrm{s}}, g_{2}^{\mathrm{s}}\right)$. Let $\left(\Delta w_{1}, \Delta w_{2}\right)$ be a redistribution of wealth such that $g_{i}+\Delta w_{i} \geqq 0$ for $i=1,2$. Then the Stackelberg equilibrium after this redistribution is $\left(g_{1}^{s}+\Delta w_{1}, g_{2}^{s}+\Delta w_{2}\right)$ and the total amount of the public good remains unchanged.

Proof. The first-order condition for the Stackelberg equilibrium is

$$
\partial u_{1}\left(g_{1}+g_{2}, w_{1}-g_{1}\right) f_{2}^{\prime}\left(w_{2}+g_{1}\right)-\frac{\partial u\left(g_{1}+g_{2}, w_{1}-g_{1}\right)}{\partial x_{1}}=0 .
$$

Let $\left(\Delta w_{i}\right)$ be a redistribution of wealth and let agent $i$ change his contribution by $\Delta g_{i}=\Delta w_{i}$ for $i=1,2$. Note that since $\Delta w_{i}+\Delta w_{2}=0$ we must have $\Delta g_{1}+\Delta g_{2}=0$. Now simply observe that if each agent changes his contribution in this way the first-order condition is still satisfied.

Warr (1983) and Bergstrom et al. (1986) show that essentially the same result holds in an (interior) Nash equilibrium. Bergstrom et al. (1986) also investigate the boundary cases in some detail. In the two-agent context we are investigating here the analysis of the boundary cases is quite straightforward so we simply state the result.

Fact 5. Suppose that person 1 is contributing and person 2 is not. Then a redistribution from 2 to 1 will increase the amount of the public good, while a redistribution from 1 to 2 can decrease or increase the amount of the public good. 


\subsection{Comparison to the Nash equilibrium}

Theorem 2. The amount of the public good contributed by agent 1 in the Stackelberg equilibrium is never larger than the amount provided by agent $I$ in the Nash equilibrium. That is, $g_{1}^{\mathrm{s}} \leqq \mathrm{g}_{1}^{\mathrm{n}}$.

Proof. There are two cases to consider: the case where $g_{2}^{\mathrm{n}}=0$, and the case where $g_{2}^{n}>0$. In the first case, it follows from Fact 3 that the Nash equilibrium is also a Stackelberg equilibrium. Hence, $g_{1}^{\mathbf{n}}=g_{1}^{\mathbf{s}}$.

As for the second case, let $V_{1}\left(g_{1}\right)=u_{1}\left(g_{1}+G_{2}\left(g_{1}\right), w_{1}-g_{1}\right)$. The derivative of $V_{1}\left(g_{1}\right)$ evaluated at the Nash equilibrium is

$$
\begin{aligned}
V_{1}^{\prime}\left(g_{1}^{\mathrm{n}}\right) & =\frac{\partial u_{1}\left(G^{\mathrm{n}}, w_{1}-g_{1}^{\mathrm{n}}\right)}{\partial G}\left[1+G_{2}^{\prime}\left(g_{1}^{\mathrm{n}}\right)\right]-\frac{\partial u_{1}\left(G^{\mathrm{n}}, w_{1}-g_{1}^{\mathrm{n}}\right)}{\partial x_{1}} \\
& =\frac{\partial u_{1}\left(G^{\mathrm{n}}, w_{1}-g_{1}^{\mathrm{n}}\right)}{\partial G} G_{2}^{\prime}\left(g_{1}^{\mathrm{n}}\right)<0 .
\end{aligned}
$$

This follows since $\partial u_{1} / \partial G-\partial u_{1} / \partial x_{1}=0$ at the Nash equilibrium and $G_{2}^{\prime}\left(g_{1}^{\text {n }}\right)$ is strictly negative when $g_{2}^{\mathrm{n}}>0$. This shows that agent 1 's utility will increase by decreasing his contribution, from which it follows that $g_{1}^{s}<g_{1}^{\text {n }}$.

Corollary. The total amount of the public good in the Stackelberg equilibrium is less than or equal to the total amount provided in the Nash equilibrium.

Proof. According to Fact 1, the function $H\left(g_{1}\right)=g_{1}+G_{2}\left(g_{1}\right)$ is an increasing function. Therefore

$$
H\left(g_{1}^{\mathrm{n}}\right)=g_{1}^{\mathrm{n}}+G_{2}\left(g_{1}^{\mathrm{n}}\right)=g_{1}^{\mathrm{n}}+g_{2}^{\mathrm{n}} \geqq g_{1}^{\mathrm{s}}+G_{2}\left(g_{1}^{\mathrm{s}}\right)=H\left(g_{1}^{\mathrm{s}}\right) .
$$

The corollary follows.

\section{Subsidy-setting mechanisms for public goods with general utilities}

Here we describe some generalizations of the two-person, subsidy-setting game described earlier. Each mechanism is a variation on the compensation mechanism described in Varian (1989).

\subsection{Subsidy setting with more than two agents}

The subsidy-setting game described earlier only works for two agents with quasilinear utility. If there are more than two agents, each agent still prefers that the other agents' contributions be subsidized - but each agent wants 
someone else to do the subsidizing. However, by adjusting who subsidizes whom, it is possible to overcome this sort of free riding in subsidy setting.

The trick is to have agent 1 set the rate at which agent 2 will subsidize agent 3's contributions. Agent 2, in turn, sets the rate at which agent 3 will subsidize l's contributions, and so on. More specifically, let $s_{j}^{i}$ be the subsidy facing agent $j$ as set by agent $i$. As before, let $x_{1}$ be agent l's private consumption, and $g_{1}$ be his contribution to the public good. If there are three agents doing the contributing, agent 1's budget constraint will be

$$
x_{1}+\left(1-s_{1}^{2}-s_{1}^{3}\right) g_{1}=w_{1}-s_{2}^{3} g_{2}-s_{3}^{2} g_{3} .
$$

Note that, unlike the two-person case, the prices agent $i$ faces are independent of the prices he sets.

In order to establish the result, we need a regularity assumption on demand functions: they must be locally invertible functions of the subsidy rates. This means that if agent 1 wants agent 2 to contribute a little more or a little less to the public good, there is some subsidy rate that he can set that will induce agent 2 to do this. Local invertibility will be satisfied if the derivative of each agent's demand function with respect to price is not zero at the equilibrium. ${ }^{3}$

Theorem 3. Let each agent have continuous convex preferences. Assume that the demand functions are locally invertible. Then the subgame perfect equilibria of the subsidy-setting game are Lindahl allocations.

Proof. We prove the theorem for three agents, but the idea extends to an arbitrary number of agents. Let $\left(x_{i}^{*}, g_{i}^{*}\right)$ be a subgame perfect equilibrium of this game, and let $\left(x_{i}^{\prime}, g_{i}^{\prime}\right)$ be an allocation that Pareto dominates it. Since preferences are convex and continuous, we can assume that $\left(x_{i}^{\prime}, g_{i}^{\prime}\right)$ is arbitrarily close to $\left(x_{i}^{*}, g_{i}^{*}\right)$.

Agent $i$ chooses his own contribution directly and chooses the other agents' contributions through the subsidy rates that he sets for them. If each agent prefers $\left(x_{i}^{\prime}, g_{i}^{\prime}\right)$ to $\left(x_{i}^{*}, g_{i}^{*}\right)$, then this allocation must not be affordable for each agent at the equilibrium subsidy rates. This yields three inequalities:

$$
\begin{aligned}
& x_{1}^{\prime}+\left(1-s_{1}^{2}-s_{1}^{3}\right) g_{1}^{\prime}>w_{1}-s_{2}^{3} g_{2}^{\prime}-s_{3}^{2} g_{3}^{\prime}, \\
& x_{2}^{\prime}+\left(1-s_{2}^{1}-s_{2}^{3}\right) g_{2}^{\prime}>w_{2}-s_{3}^{1} g_{3}^{\prime}-s_{1}^{3} g_{1}^{\prime}, \\
& x_{3}^{\prime}+\left(1-s_{3}^{1}-s_{3}^{2}\right) g_{3}^{\prime}>w_{3}-s_{2}^{1} g_{2}^{\prime}-s_{1}^{2} g_{1}^{\prime} .
\end{aligned}
$$

Adding these inequalities gives

\footnotetext{
${ }^{3}$ This is automatically satisfied if utility is quasilinear and strictly concave.
} 


$$
\sum_{i=1}^{3} x_{i}^{\prime}+\sum_{i=1}^{3} g_{i}^{\prime}>\sum_{i=1}^{3} w_{i},
$$

which shows that the Pareto-dominating allocation is not feasible.

Next we show that the equilibrium is Lindahl. Agent 1 can choose $g_{1}$ directly and choose $g_{2}$ or $g_{3}$ indirectly, through the subsidy he sets for the other agents. Since the contributions are perfect substitutes in consumption, they must have the same equilibrium price. Hence we can define $p_{1}=$ $1-s_{1}^{2}-s_{1}^{3}=s_{3}^{2}=s_{2}^{3}$. From this it follows that agent 1 's budget constraint can be written as

$$
x_{1}+p_{1}\left(g_{1}+g_{2}+g_{3}\right)=x_{1}+p_{1} G=w_{1},
$$

which shows that the allocation is Lindahl.

It is worth observing that this mechanism is balanced both in and out of equilibrium. The proof that the equilibrium is Lindahl is essentially the proof given in Varian (1989) for general externalities, specialized to the case of public goods.

\subsection{Another mechanism for implementing Lindahl allocations}

Here is another two-stage mechanism that implements Lindahl allocations. The message space in this mechanism is much smaller than in the previous mechanism since each agent has to name only a single number.

(a) The price-setting stage. Each agent $i$ announces a number $q_{i}$. The price for agent $i$ 's contribution to the public good is the average of the numbers named by the other agents:

$$
p_{i}=\frac{1}{n-1} \sum_{j \neq i} q_{j} .
$$

(b) The contribution stage. Each agent $i$ chooses $\left(x_{i}, g_{i}\right)$ to maximize his utility subject to the budget constraint

$$
x_{i}+p_{i} g_{i}=w_{i}-p_{i} \sum_{j \neq i} g_{j}-Q(p)
$$

where the penalty term is given by $Q(p)=\left(\sum_{j=1}^{n} p_{j}-1\right)^{2}$.

In the second stage we have each agent choosing a contribution to the public good for which he pays a price $p_{i} g_{i}$. But agent $i$ must also make a payment based on the amount of the good contributed by the other agents. Since the total amount of the public good is $G=g_{i}+\sum_{j \neq i} g_{j}$, we could also write agent $i$ 's budget constraint as $x_{i}+p_{i} G=w_{i}-Q(p)$. 
It is easy to show that the Lindahl allocation $\left(p_{i}^{e}, G^{\mathrm{e}}\right)$ is an equilibrium of this game. To do this we only need to show that if every agent makes announcements that lead to the Lindahl prices, agent $i$ cannot increase his utility by announcing something that yields non-Lindahl prices for the other agents.

To prove this we first observe that since the Lindahl prices $\left(p_{i}^{e}\right)$ result in an efficient amount of the public good; the penalty term, $Q(p)$, must be zero by the standard Samuelson efficiency condition. ${ }^{4}$ Suppose that agent $i$ announces some numbers that change the price vector $p^{e}$ to $p^{\prime}$ and that this change results in some possibly different amount of the public good, $G^{\prime}$. Note that agent $i$ can only affect the prices facing the other agents, not the price he faces.

We have

$$
u_{i}\left(G^{\mathrm{e}}, w_{i}-p_{i}^{\mathrm{e}} G^{\mathrm{e}}\right) \geqq u_{i}\left(G^{\prime}, w_{i}-p_{i}^{\mathrm{e}} G^{\prime}\right) \geqq u_{i}\left(G^{\prime}, w_{i}-p_{i}^{\mathrm{e}} G^{\prime}-Q\left(p^{\prime}\right)\right) .
$$

The first inequality comes from the fact that we start with a Lindahl allocation; the second inequality comes from the fact that $Q\left(p^{\prime}\right) \geqq 0$. This argument shows that agent $i$ is at least as well off announcing prices that lead to the Lindahl prices $\left(p_{j}^{\mathrm{e}}\right)$ as any other prices; i.e. that Lindahl allocations are an equilibrium to the mechanism. In the appendix I show that there can be no other equilibria of the mechanism.

I have described this mechanism in terms of setting prices. However, it can also be described in terms of setting subsidies, which makes it look more like the mechanisms described earlier. In the subsidy-setting framework, each agent $i$ names a number $1-s_{i}$ which turns out in equilibrium to be the rate at which agent $i$ 's contributions are subsidized and the rate at which agent $i$ subsidizes everyone else's contributions. We denote the contributions by everyone except agent $i$ by $G_{-i}=\sum_{j \neq i} g_{j}$. The budget constraint facing agent $i$ then can be written as

$$
\left(1-\sum_{j \neq i} s_{j}\right) g_{i}+x_{i}=w_{i}-\left(1-\sum_{j \neq i} s_{j}\right) G_{-i}-\left(1-\sum_{j=1}^{n} s_{j}\right)^{2} .
$$

It can be shown that in equilibrium $s_{i}+\sum_{j \neq 1} s_{j}=1$, so that agent $i$ ends up being subsidized at the rate $1-s_{i}$ and subsidizing the other agents at the same rate.

\subsection{Related literature}

Danziger and Schnytzer (1991) have independently examined a subsidy-

\footnotetext{
${ }^{4}$ We assume that it is efficient to provide a positive amount of the public good.
} 
setting mechanism similar in spirit to this one. In the Danziger-Schnytzer mechanism, each agent names a rate at which he will subsidize the other agents' contributions. In my mechanism, the rate at which each agent subsidizes the other agents is set by the other agents. Furthermore, my mechanism requires a penalty function while a penalty is not necessary in the Danziger-Schnytzer mechanism. The Danzigner-Schnytzer mechanism is therefore a bit simpler in the case of pure public goods. However, I show in Varian (1989) that my mechanism also works for general externalities problems.

\section{Incomplete information}

Until now we have examined games where each agent knows the preferences and wealth of the other agent. Here we consider a model where each contributor has incomplete information about the other contributor. In our game the second contributor reacts passively, making his optimal choice given the first agent's contribution. Hence it is irrelevant whether or not he knows anything about the first contributor. The only interesting uncertainty concerns the first contributor's knowledge of the second contributor's preferences.

Consider the quasilinear model examined earlier. In this case all that is relevant from the first contributor's point of view is the value of $\bar{g}_{2}$ - how much the second person will contribute if the first person contributes zero. Suppose that the first contributor has a prior distribution on how much the other person will contribute and seeks to maximize expected utility. ${ }^{5}$

The expected utility of the first contributor is

$$
\begin{aligned}
V_{1}\left(g_{1}\right) & =\int_{0}^{\infty}\left[u\left(g_{1}+\max \left\{\bar{g}_{2}-g_{1}, 0\right\}\right)-g_{1}\right] f\left(\bar{g}_{2}\right) \mathrm{d} \bar{g}_{2} \\
& =\int_{0}^{g_{1}}\left[u\left(g_{1}\right)-g_{1}\right] f\left(\bar{g}_{2}\right) \mathrm{d} \bar{g}_{2}+\int_{g_{1}}^{\infty}\left[u\left(\bar{g}_{2}\right)-g_{1}\right] f\left(\bar{g}_{2}\right) \mathrm{d} \bar{g}_{2} \\
& =\left[u\left(g_{1}\right)-g_{1}\right] F\left(g_{1}\right)+\int_{g_{1}}^{\infty}\left[u\left(\bar{g}_{2}\right)-g_{1}\right] f\left(\bar{g}_{2}\right) \mathrm{d} \bar{g}_{2} .
\end{aligned}
$$

Differentiating this expression with respect to $g_{1}$ and simplifying yields

$$
V_{1}^{\prime}\left(g_{1}\right)=u_{1}^{\prime}\left(g_{1}\right) F\left(g_{1}\right)-1 .
$$

Note that when $g_{1}=0$, the probability that $\bar{g}_{2}$ is less than $g_{1}$ is zero, so that

\footnotetext{
${ }^{5}$ We assume that the von Neumann-Morgenstern utility function takes the quasilinear form. This is restrictive, but seems necessary for a simple analysis.
} 
$V_{1}^{\prime}(0)=-1$. If $g_{1}$ is large enough so that agent 1 is certain that $\bar{g}_{2}$ is less than $g_{1}$, then $V_{1}^{\prime}\left(g_{1}\right)=u_{1}^{\prime}\left(g_{1}\right)-1$. Hence, agent 1 's utility as a function of his gift is similar to the shape depicted in fig. 2. Depending on the beliefs of agent 1 about agent 2's maximum contribution, agent 1 will either choose to free ride, or to contribute an amount $g_{1}^{*}$ that satisfies the condition

$$
u_{1}^{\prime}\left(g_{1}^{*}\right) F\left(g_{1}^{*}\right)-1=0 .
$$

This marginal condition is quite intuitive. If agent 1 decides to contribute a bit more of the public good, he will get $u_{1}^{\prime}\left(g_{1}^{*}\right)$, but only if agent 2 has $\bar{g}_{2}<g_{1}^{*}$. Otherwise, agent 1 will get no incremental utility from his contribution - since his contribution would just crowd out some of the public good that agent 2 would have contributed anyway. Hence the expected marginal utility of agent 1's contribution is $u_{1}^{\prime}\left(g_{1}^{*}\right)$ times the probability that $\bar{g}_{2}<g_{1}^{*}$, which is just $u_{1}^{\prime}\left(g_{1}^{*}\right) F\left(g_{1}^{*}\right)$. The optimal contribution is determined by the condition that this expected marginal utility must equal the (certain) marginal cost of the contribution.

How does this amount compare with $\bar{g}_{1}$, which is what agent 1 would contributc under certainty? Notc that $V_{1}^{\prime}\left(\bar{g}_{1}\right)=u_{1}^{\prime}\left(\bar{g}_{1}\right) F\left(\bar{g}_{1}\right)-1=F\left(\bar{g}_{1}\right)-1$. As long as there is some possibility that agent 2 will have $\bar{g}_{2}>\bar{g}_{1}$, we will have $F\left(\bar{g}_{1}\right)<1$ and $V_{1}^{\prime \prime}\left(\bar{g}_{1}\right)$ will be negative. If $V_{1}\left(g_{1}\right)$ is concave, this implies that the equilibrium contribution in the presence of uncertainty is less than the contribution under certainty. Intuitively, the possibility that agent 2 may value the good more than agent 1 leads agent 1 to reduce his contribution to the public good, hoping to free ride on agent 2's contribution.

\section{Summary}

We have examined some sequential games involving contributions to a public good. If preferences are quasilinear, then:

(1) The sequential equilibrium of the contribution game will provide the same or less of the public good than the simultaneous-move game.

(2) The player who likes the public good least will bid the most to move first.

(3) Each player would like to subsidize the other player's contributions. If both players choose subsidy rates and then play the voluntary contribution game, a Lindahl equilibrium is the unique subgame perfect equilibrium of this two-stage game.

(4) The equilibrium of the sequential-move game is independent of small redistributions of wealth.

(5) If the first agent is uncertain about the type of the second agent, he will tend to contribute less to the public good.

In the case of general utility functions, the amount of the public good 
supplied in the Stackelberg game will never be more than in the Nash game. Furthermore, there are several generalizations of the subsidy-setting game that yield Lindahl allocations.

\section{Appendix: Proof of uniqueness in the price-setting contribution game}

Here we provide a proof of the claim made in the text that the only equilibria of our price-setting mechanism are Lindahl allocations. We assume that for some agent the marginal rate of substitution between the private and the public good is infinite when $G=0$ so that someone will always want to make a positive contribution to the public good. We also assume local invertibility of the sort described earlier: there is a set of prices that will result in any desired (small) change in behavior. See Varian (1989) for more discussion of the role of this assumption.

Let $\left(p_{i}^{*}\right)$ be the set of prices that results from the equilibrium announcements $\left(q_{i}^{*}\right)$. We first show that in equilibrium we must have $\sum_{k=1}^{n} p_{k}^{*}=1$. Suppose not. We have assumed that at least one agent $i$ will choose a positive amount of the public good. Suppose that this agent changes his announcement, $q_{i}$. This will change agent $i$ 's utility by

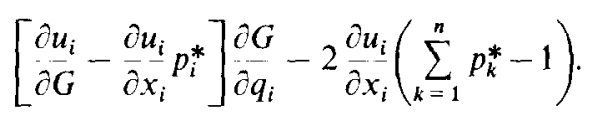

Since agent $i$ is contributing a positive amount the bracketed expression vanishes by the envelope theorem, leaving us with a term that is, by assumption, non-zero. Hence there is some change in i's announcement that will increase his utility, contradicting the assumption of equilibrium. It follows that $\sum_{k=1}^{n} p_{k}^{*}=1$ in equilibrium.

Now suppose that the equilibrium allocation is not Pareto efficient. Then there is some other feasible allocation $\left(x_{i}^{\prime}, G^{\prime}\right)$ that all agents prefer. By continuity and convexity, we can take this allocation to be arbitrarily close to the equilibrium allocation. Local invertibility implies that there is some announcement of prices that each agent can make that will implement this dominating allocation. If they choose not to do so, it must be because the dominating allocation violates their budget constraint,

$$
x_{i}^{\prime}+p_{i} G^{\prime}>w_{i} \text {. }
$$

Summing over the agents and using the fact that $\sum_{k=1}^{n} p_{k}^{*}=1$, we have

$$
\sum_{i} x_{i}^{\prime}+G^{\prime}>\sum_{i} w_{i}
$$

which contradicts the assumption that the dominating allocation is feasible. 


\section{References}

Admati, A. and M. Perry, 1991, Joint projects without commitment, Review of Economic Studies 58, 259-276.

Bergstrom, T., 1989, Love and spaghetti, the opportunity cost of virtue, Journal of Economic Perspectives 3, 165-173.

Bergstrom, T., L. Blume and H. Varian, 1986, On the private provision of public goods, Journal of Public Economics 29, 25-49.

Buchanan, J.M., 1975, The Samaritan's dilemma, in: E.S. Phelps, ed., Altruism, morality, and economic theory (The Sage Foundation, New York).

Cornes, R. and T. Sandler, 1986, The theory of externalities, public goods, and club goods (Cambridge University Press, Cambridge, U.K.).

Danziger, L. and A. Schnytzer, 1991, Implementing the Lindahl voluntary-exchange mechanism, European Journal of Political Economy 7, 55-64.

Forsyth, A., 1986, A natural history of sex (Charles Scribner's Sons, New York).

Groves, T., 1979, Efficient collective choice when compensation is possible, Review of Economic Studies 46, 227-241.

Groves, T. and J. Ledyard, 1977, Optimal allocations of public goods: A solution to the 'free rider problem', Econometrica 45, 783-809.

Groves, T. and J. Ledyard, 1987, Incentive compatibility since 1972, in: T. Groves, R. Radner and S. Reiter, eds., Information, incentives and economic mechanisms (University of Minnesota Press, Minneaopolis, MN).

Guttman, J., 1978, Understanding collective action: Matching behavior, American Economic Review 68, 251-255.

Guttman, J., 1986, Matching behavior and collective action: Some experimental evidence, Journal of Economic Behavior and Organization 7, 171-198.

Guttman, J., 1987, A non-Cournot model of voluntary collective action, Economica 54, 1-19.

Hurwicz, L., 1979, Outcome functions yielding Walrasian and Lindahl allocations at Nash equilibrium points, Review of Economic Studies 46, 217-225.

Jackson, M. and H. Moulin, 1992, Implementing a public project and distributing its cost, Journal of Economic Theory 57, 125-140.

Ledyard, J., 1992, Public goods: A survey of experimental research, Working Paper, California Institute of Technology.

Lindbeck, A. and L.W. Weibull, 1988, Altruism and time consistency: The economics of fait accompli, Journal of Political Economy 96, 1165-1182.

Moore, J., 1991, Implementation in environments with complete information, Working Paper, Suntory-Toyota International Centre for Economics, London School of Economics.

Roberts, R., 1987, Financing public goods, Journal of Political Economy 95, 420-437.

Varian, H., 1982, Pensions and public policy, Working Paper, University of Michigan.

Varian, H., 1989, A solution to the problem of externalities when agents are well informed, Working Paper, University of Michigan.

Walker, M., 198!, A simple incentive compatible scheme for attaining Lindahl allocations, Econometrica 48, 56-73.

Warr, P. 1983, The private provision of a public good is independent of the distribution of income, Economics Letters 13, 207-211. 